\title{
Correction to: Impact of Motivation on Selected Aspects of Attention in Children with ADHD
}

\author{
Sebastian Skalski ${ }^{1}\left[\right.$ [D $\cdot$ Grzegorz Pochwatko $^{1}$ ([) $\cdot$ Robert Balas $^{1}$ (])
}

Published online: 5 November 2020

○) Springer Science+Business Media, LLC, part of Springer Nature 2020

\section{Correction to: Child Psychiatry \& Human Development https://doi.org/10.1007/s10578-020-01042-0}

The original online version of this article was revised due to the omission of "Objective of the Study" at the end of Introduction section.

\section{Objective of the Study}

The purpose of this study was to assess the impact of extrinsic motivation on selected aspects of attention in children with ADHD, as well as to measure cortical activity and dimensions of motivation as per the self-determination theory.

Based on the aforementioned articles, we assumed that: (Hypothesis 1) children with ADHD may exhibit lower levels of cortical activity as measured using (a) TBR in the midline and the (b) HEG ratio in prefrontal areas than TD children; (H2) children with ADHD may display lower levels of (a) intrinsic motivation and (b) identified regulation as well as higher levels of (c) external regulation, (d) introjected regulation and (e) amotivation to learning compared to TD children; (H3) children with ADHD may exhibit deficits regarding: (a) vigilance, (b) visual search and (c) divided attention; (H4) increasing the motivation may reduce attention deficits in children with ADHD with regard to: (a) vigilance, (b) visual search and (c) divided attention. The obtained data will enable a better understanding of ADHD issues.

The original article can be found online at https://doi.org/10.1007/ s10578-020-01042-0.

Sebastian Skalski

sebastian.skalski@sd.psych.pan.pl

1 Institute of Psychology, Polish Academy of Sciences, 1

Jaracza Street, 00-378 Warsaw, Poland
Additionally, there are different names for theta/beta power ratio and HEG ratio once from lowercase and then from uppercase. These names should be display as lowercase everywhere.

Publisher's Note Springer Nature remains neutral with regard to jurisdictional claims in published maps and institutional affiliations. 